\title{
Challenges in Measuring External Currents Driven by the Solar Wind-Magnetosphere Interaction
}

\author{
Guan Le ${ }^{1, *}$, James A. Slavin ${ }^{2}$, and Robert F. Pfaff ${ }^{1}$ \\ ${ }^{1}$ Space Weather Laboratory, Heliophysics Science Division, NASA Goddard Space Flight Center, Greenbelt, Maryland, USA \\ ${ }^{2}$ Department of Atmospheric, Oceanic and Space Sciences, University of Michigan, Ann Arbor, Michigan, USA
}

Received 31 May 2013, revised 13 February 2014, accepted 19 August 2014

\begin{abstract}
In studying the Earth's geomagnetism it has always been a challenge to separate the external currents originating from the ionosphere and magnetosphere. While the internal magnetic field changes very slowly in time scales of years and more, the ionospheric and magnetospheric current systems driven by the solar wind-magnetosphere interaction are very dynamic. They are intimately controlled by the ionospheric electrodynamics and ionosphere-magnetosphere coupling. Single spacecraft observations are not able to separate their spatial and temporal variations, and thus to accurately describe their configurations. To characterize and understand the external currents, satellite observations require both good spatial and temporal resolutions. This paper reviews our observations of the external currents from two recent Low Earth Orbit (LEO) satellite missions: Space Technology 5 (ST-5), NASA's first three-satellite constellation mission in LEO polar orbit and Communications/Navigation Outage Forecasting System (C/NOFS), an equatorial satellite developed by the US Air Force Research Laboratory. We present recommendations for future geomagnetism missions based on these observations.
\end{abstract}

Key words: Geomagnetism, Solar wind-magnetosphere interaction, Ionospheric currents, Magnetospheric currents

Citation: Le, G., J. A. Slavin, and R. F. Pfaff, 2015: Challenges in measuring external currents driven by the solar wind-magnetosphere interaction. Terr. Atmos. Ocean. Sci., 26, 11-25, doi: 10.3319/TAO.2014.08.19.02(GRT)

\section{INTRODUCTION}

The existence of the Earth's internal magnetic field is vital to life on Earth because it acts as a giant shield to protect the Earth from the solar wind (charged particles from the sun) and cosmic rays. The Earth's main magnetic field is generated by an internal electric current maintained by a rotating and electrically conducting fluid in the Earth's outer core powered by the convective geodynamo (Glatzmaier and Roberts 1995a, b). Studying geomagnetism by measuring and monitoring the Earth's magnetic field provides an important way to probe the Earth's liquid core and its change with time. However, other sources of magnetisms, although small in comparison with the main field from the internal source, also contribute to the Earth's magnetic field. They include crustal magnetic fields, ocean currents and external currents originating from the ionosphere and the magnetosphere. At any location and any moment the magnetic field is the vector sum of the fields from all of these sources.

\footnotetext{
* Corresponding author

E-mail:Guan.Le@nasa.gov
}

External currents originating from the ionosphere and the magnetosphere contaminate the geomagnetism magnetic field measurements. It has always been a big challenge to separate the magnetic field from internal and external sources in studying the Earth's geomagnetism. Although there is a long history of direct ground measurements of the Earth's magnetic field, ground-based observations suffer from uneven spatial coverage and large gaps in the oceans. Only Low Earth Orbit (LEO) satellites can provide true global mapping of the Earth's magnetic field. High precision measurements from dedicated geomagnetism satellites such as Magsat (1979 - 1980), Orsted (since 1999), and CHAMP (2000 - 2010) have resulted in significant advances in monitoring, modeling, and understanding the Earth's magnetic field (see Olsen and Stolle 2012 for a review).

The geomagnetic field changes at various time scales. The internal magnetic field changes very slowly, in time scales of years and more. However, the external currents are very dynamic and vary over much shorter time scales (seconds to days). To characterize and understand the external 
currents, satellite observations require both good spatial and temporal resolutions. Single spacecraft measurements do not allow us to separate spatial and temporal variations, and thus are unable to accurately and fully describe their configurations. This paper will discuss our recent LEO spacecraft observations of the external currents driven by the solar windmagnetosphere interaction and make recommendations for future geomagnetism missions.

\section{EXTERNAL CURRENT SYSTEM DRIVEN BY THE SOLAR WIND-MAGNETOSPHERE INTERACTION}

To a first order approximation the Earth's internal magnetic field is dipolar resembling that of a bar magnet. It acts as an effective obstacle to the flow of charged particles from the sun, called the solar wind. The interaction with the solar wind flow confines the Earth's magnetic field within a cavity called the magnetosphere by compressing the dayside and stretching the nightside magnetic field lines. The magnetospheric cavity has a compressed dayside and a long cometlike tail, which is significantly distorted from the dipolar magnetic field. Such a distortion and the overall shape of the magnetosphere are the direct result of the presence of largescale electric current systems in the magnetosphere and the ionosphere that are driven by the solar wind-magnetosphere interaction. Figure 1 illustrates the large-scale electric current systems in the magnetosphere and ionosphere. They include: the magnetopause (Chapman-Ferraro) current flowing on the magnetosphere boundary, the ring current in the inner magnetosphere, the tail current flowing in the neutral sheet across the magnetotail, field-aligned (Birkeland) currents flowing in and out of the ionosphere and coupling the magnetosphere to the ionosphere, as well as associated horizontal currents in the ionosphere. The horizontal ionospheric currents include
Pedersen currents in the auroral zone and across the polar cap, and auroral electrojets (Hall currents) around the auroral oval. These magnetospheric and ionospheric currents respond dynamically to variations in the solar wind plasma and the interplanetary magnetic field (IMF). Changes in these current systems cause geomagnetic disturbances. Thus, the solar wind-magnetosphere interaction is the main driver for various geomagnetic activities over short time scales (seconds to days). The ultimate energy source is provided by the solar wind from the Sun's atmosphere. The term "space weather" is used to refer to the changing environment of plasma, magnetic fields and radiation in near-Earth and interplanetary space due to solar variability. Large-scale electric currents in the magnetosphere and ionosphere constitute important space weather parameters. During magnetic storms and substorms, these currents intensify in response to the enhanced solar wind-magnetosphere interaction.

In the region below $\sim 1000 \mathrm{~km}$ from the surface of the Earth, where geomagnetism satellites fly, the external currents that generate the largest magnetic fields in contaminating the geomagnetism measurements are field-aligned currents (FACs) at auroral latitudes, horizontal currents in the high latitude ionosphere, and the ring current in the inner magnetosphere. Among them, FACs flow into and out of the ionosphere in the auroral zone, and are closed by horizontal Pedersen currents to complete the current loops in the auroral zone and across the polar cap in the ionosphere. The combined FAC-Pedersen current loops are mostly invisible on the ground because the magnetic fields are confined within the current loops. However, polar orbiting LEO satellites pass right through the FAC layers and make direct in situ measurements of the magnetic field disturbances generated by the combined FAC-Pedersen current loops. Their magnetic field perturbations are transverse to the background magnetic field, and can reach to over $1000 \mathrm{nT}$ in the
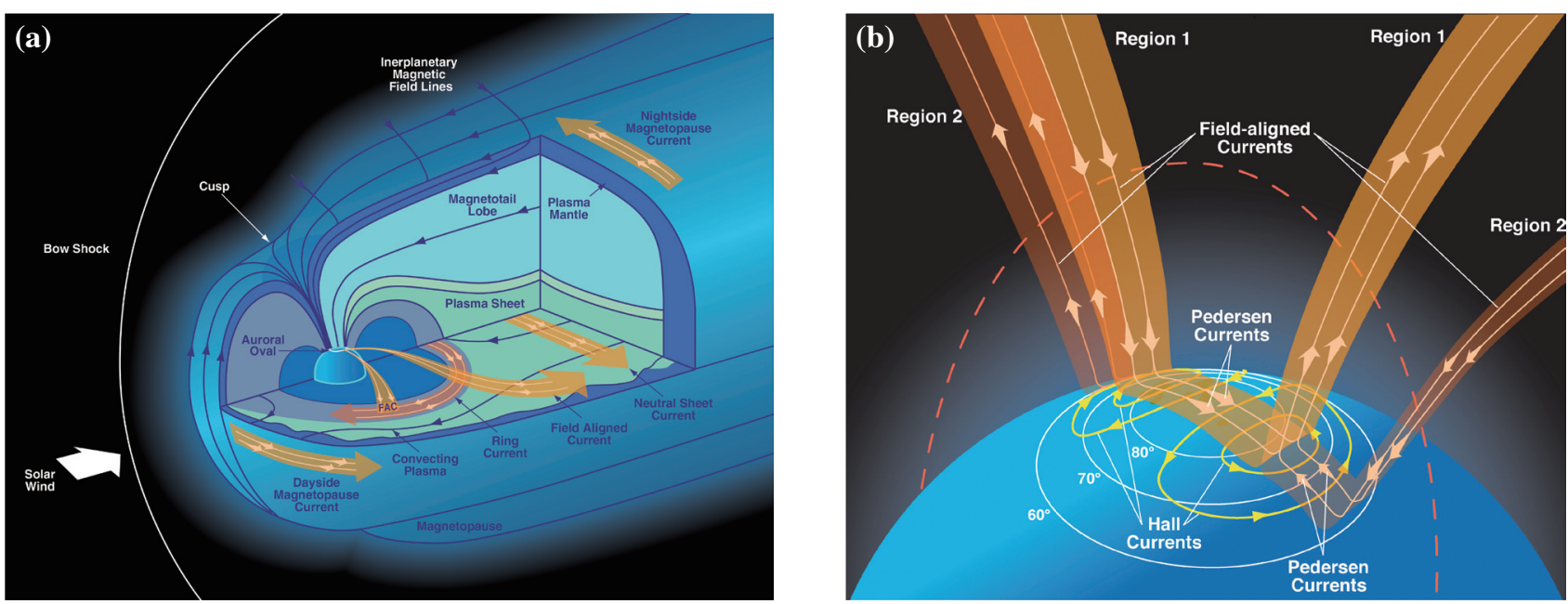

Fig. 1. Illustration of large-scale electric current systems in the magnetosphere and the ionosphere. (a) Magnetospheric current systems; (b) Ionospheric current systems (modified from Le et al. 2010). 
magnetic field components. There is no perturbation in the magnetic field strength in the in-situ measurements because the transverse magnetic field perturbation only twists the magnetic field lines without changing the field strength. Auroral electrojets (Hall currents) are another type of large-scale horizontal current in the high latitude ionosphere. They flow in the auroral oval, westward in the dawn side and eastward in the duskside. They are largely closed within themselves in the polar ionosphere. During substorms, enhanced westward auroral electrojets, called the substorm current wedge are fed by FACs from disrupted tail current (McPherron et al. 1973). The magnetic field signatures of auroral electrojets can be readily measured on the ground below the auroral zone (e.g., Kamide et al. 1981; Friis-Christensen et al. 1985). In space, auroral electrojets flow below LEO satellites. They cause a magnetic perturbation mainly in the magnetic field strength in auroral latitudes, either positive or negative depending on the local time (Zanetti et al. 1984), which decreases with the altitude and can be detected at altitudes below $\sim 700 \mathrm{~km}$ (Moretto et al. 2002; Le et al. 2009).

In the magnetosphere the ring current, tail current and the magnetopause current are all remote current systems to LEO satellites. They all produce global magnetic disturbances that can be readily measured on the ground and by LEO satellites. However, the magnetic fields associated with the magnetopause current and the tail currents are relatively small because they are more remote. The ring current in the inner magnetosphere makes the most significant contribution to these global disturbances due to its relative proximity to the Earth. The ring current is formed by charged particles in the magnetosphere that are trapped in the Earth's magnetic field from the solar wind through enhanced solar wind-magnetosphere interaction. It flows westward in the equatorial magnetosphere and produces a global southward magnetic field perturbation at the Earth. Since the Earth's main magnetic field is northward in the equatorial region, the ring current causes a global depression in the magnetic field strength and the equatorial average value of which, the Dst index, is used to monitor and characterize the ring current. It has been shown that the absolute value of the Dst index is proportional to the total energy content of the charged particles in the ring current region (Dessler and Parker 1959; Sckopke 1966). A prolonged negative Dst index is an indication of a magnetic storm in progress and also a measure of the storm intensity. The more negative the Dst index is, the more intense the magnetic storm. Geomagnetic storms are classified based on the Dst index, as moderate (Dst $>-100 \mathrm{nT}$ ), intense $(-250 \mathrm{nT}<$ Dst $<-100 \mathrm{nT})$ and super-storm (Dst < -250 nT). During the solar cycle 23 (May 1996 - November 2008), 11 super-storms occurred (Echer et al. 2008).

Both the magnetopause current and the tail current add to the magnetic disturbances at the Earth and contribute to the Dst index, although to a much lesser extent. The magnetopause current is the boundary of the Earth's magnetic field. It flows from dawn to dusk on the dayside, opposite to the ring current. It is controlled by the solar wind dynamic pressure and contributes a northward magnetic field of $\sim+20 \mathrm{nT}$ at the Earth and a positive value of the same amount in the Dst index during nominal solar wind conditions (Burton et al. 1975). The Dst index also shows a +20 - $30 \mathrm{nT}$ sudden rise, the so-called the storm sudden commencement (SSC) in response to a sudden increase in the solar wind dynamic pressure at the beginning of a classic magnetic storm (Dessler et al. 1960). The tail current, on the other hand, flows from dawn to dusk on the night side in the same sense of the ring current. Its contribution to the Dst index can be significant during storms and substorms. Observations show that the tail current can account for $\sim 20$ - 25\% of the measured Dst index variation during storms and substorms (Turner et al. 2000; Ohtani et al. 2001).

Although the Dst index is an indication of the ring current strength, it does not provide any information about the local time asymmetry of the ring current. Both ground-based and in situ satellite observations have provided evidence that the ring current has both symmetric and asymmetric parts, especially during stormtime (Fukushima and Kamide 1973; Greenspan and Hamilton 2000; Iyemori 2000; Turner et al. 2001). A significant fraction of the ring current is partial, which flows only within a limited longitudinal region and must be diverted out of the equatorial region as FACs to close in the ionosphere. The ring current distributions deduced from in situ magnetic field data show that the partial current is much stronger than the symmetric current, up to a factor of 5 under moderate storm conditions (Le et al. 2004). Thus, the partial ring current makes the major contribution to the Dst index. To describe the asymmetric nature of the ring current, a new set of geomagnetic disturbance indices, longitudinal asymmetric (ASY) and symmetric (SYM) indices, are introduced for both the $\mathrm{H}$ and $\mathrm{D}$ components of the magnetic field at the surface of the Earth at mid-latitude (Iyemori et al. 1992). The SYM-H is essentially the same as the hourly Dst index but with higher resolution, which is the average disturbance at every minute for the $\mathrm{H}$-components of all stations. The partial ring current contributes to the SYM-H index the same way as it does to the Dst index. Thus, SYM-H does not represent the strength of the symmetric ring current, but the average ring current strength for both the symmetric and asymmetric components. On the other hand, the ASY-H index, which is the range between the maximum and minimum deviation of the H-components from the SYM-H, is an indication of how asymmetric the ring current is.

In summary, external currents in the magnetosphere and ionosphere are very dynamic and respond to the solar wind-magnetosphere interaction. Separating these external currents from geomagnetism measurements requires characterizing their strength, spatial variation and temporal evolution for both quiet and disturbed times. The main goals of external current investigations are to understand how they 
vary with solar wind parameters, how they vary with location and local time and how they change with time. While a single polar-orbiting LEO satellite covers all latitudes for two local times once every 90 minutes, it cannot separate spatial and temporal variations. It is desirable to have significantly denser coverage in space and time with a multisatellite constellation. The ESA's magnetic field mission Swarm will be the first constellation of satellites for geomagnetism and is expected to lead to new insight into many natural processes responsible for the Earth's magnetic field, including the solar wind-magnetosphere interaction (FriisChristensen et al. 2006). In the following section, we will review our recent observations of external currents from two LEO satellite missions. One of the missions is Space Technology 5 (ST-5), NASA's first three-satellite constellation mission in LEO polar orbit as shown in Fig. 2 (Slavin et al. 2008). The other one is Communications/Navigation Outage Forecasting System (C/NOFS), an equatorial satellite developed by the US Air Force Research Laboratory (de La Beaujardière et al. 2004, 2009). Although these satellites were not equipped with instruments for geomagnetism purposes, they all carried research-quality magnetometers for studying the external currents in the ionosphere and the magnetosphere. These two missions have provided us with important magnetic field data for understanding the timespace characteristics of the external currents and valuable lessons for designing post-Swarm geomagnetism missions.

\section{ST-5 OBSERVATIONS OF FIELD-ALIGNED CURRENTS AND IONOSPHERIC CURRENTS}

ST-5 is a three micro-satellite constellation deployed into an elliptical (300 km perigee and $4500 \mathrm{~km}$ apogee), dawn-dusk, sun-synchronous polar orbit from 22 March to 21 June 2006, for technology validations. The three spacecraft are maintained in a string-of-pearl constellation with controlled spacing ranging from under $50 \mathrm{~km}$ up to $\sim 5000 \mathrm{~km}$ (ref. Fig. 1 in Slavin et al. 2008). Each spacecraft carries a boom-mounted miniature tri-axial fluxgate magnetometer, returning high quality magnetic field data as the constellation flies in formation, making simultaneous multi-point measurements of the magnetic field through the Earth's dynamic ionospheric current systems. A substantial volume of magnetic field data was taken over a range of inter-satellite spacing. These separations allow us to determine the FAC properties and separate the spatial versus temporal structures of auroral FACs over a wide range of spatial $(\sim 50-4000 \mathrm{~km})$ and temporal $(\sim 5-600 \mathrm{~s})$ scales.

FACs usually appear as quasi-planar "sheets" that tend to be loosely parallel to lines of constant geomagnetic latitude (e.g., Iijima and Potemra 1978). Typically, there is a set of "Region 1" or "R1" FACs along the high latitude edge of the auroral oval, which originate near the equatorial edge of the magnetosphere. The R1 currents flow into the ionosphere on the dawnside and out of the ionosphere on the duskside. At the lower latitude edge of the auroral oval there is also a set of "Region 2" or "R2" FACs with polarities opposite to $\mathrm{R} 1$, which originate in the region where the ring current has a divergence due to the existence of a partial ring current. The interaction between the solar wind and the magnetosphere is controlled by the IMF and solar wind conditions (e.g., Cowley 1984). The IMF and solar wind constantly change, making the FAC systems highly dynamic. Temporal variability in the FACs at time scales less than the orbit period of LEO spacecraft ( $\sim 90 \mathrm{~min}$ ) cannot be assessed using data from a single spacecraft. The data from ISEE 1 and 2 magnetometers provided the first dualpoint simultaneous measurements of FACs at mid-altitudes (2.4 - $7 \mathrm{R}_{\mathrm{E}}$ ). The four-spacecraft Cluster data were also used to study FACs at mid- and high-altitudes $\left(4-11 \mathrm{R}_{\mathrm{E}}\right.$ ) (e.g., Cargill et al. 2001; Johansson et al. 2004; Draper et al. 2005; Figueiredo et al. 2005). The 3 -spacecraft ST5 mission provides the first multi-point measurements of FACs at low altitudes $(\sim 300-4500 \mathrm{~km})$, which are complementary to the mid- and high-altitude observations.

\subsection{The Current Density, Motion and Velocity of FACs}

Previously the standard method for calculating the current density from single spacecraft magnetic field data requires the assumption that the FAC is a stationary, infinite current sheet to the east-west direction (Iijima and Potemra 1976). As the spacecraft passes through the stationary current sheet, the magnetic field perturbation in the eastward component $\delta B_{E}$ and the spacecraft velocity in the northward direction $V_{S / C}$ (same as the current sheet normal) are used to calculated the current density:

$$
J_{/ /}=\left(-1 / \mu_{0}\right)\left(1 / V_{S / C}\right)\left(\partial B_{E} / \partial t\right)
$$

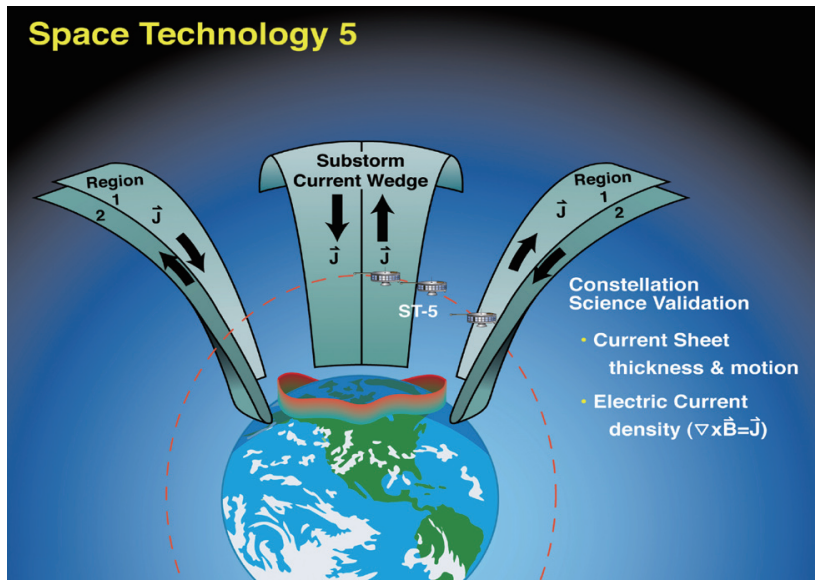

Fig. 2. Overview of Space Technology 5 (ST-5), NASA's first threesatellite constellation mission in LEO polar orbit (adapted from Slavin et al. 2008) 
Similarly, the thickness of the current sheet can be determined as $L=V_{S / C} \cdot \delta t$, where $\delta t$ is the time duration of the current sheet crossing. The errors in such calculations are positively correlated to the ratio of $\left|V_{C S} / V_{S / C}\right|$, where $V_{C S}$ is the northward velocity component of the current sheet motion. The simultaneous multi-point measurements from ST-5 constellation allow us to determine the velocity of the current sheet motion and thus to relax the assumption that the current sheet is stationary. From the spacecraft locations and the times when two spacecraft observe the same current sheet structure, we can determine $V_{C S}$. The current density can then be determined more accurately by correcting for the motion of the current sheet (Slavin et al. 2008):

$J_{/ /}=\left(-1 / \mu_{0}\right)\left[1 /\left(V_{S / C}-V_{C S}\right)\right]\left(\partial B_{E} / \partial t\right)$

A statistical study using the entire ST-5 data set shows that the current sheet velocity is quite variable and occurs in a large range from -1 to $1 \mathrm{~km} \mathrm{~s}^{-1}$ at ST-5 altitudes of $\sim 300-5000 \mathrm{~km}$; and current sheets tend to move faster/ slower during intervals of higher/lower geomagnetic activities (Wang et al. 2009). The ratio $\left|V_{C S} / V_{S / C}\right|$ occurs in the range $\sim 0-25 \%$ with the median (mean) value of $4 \%(6 \%)$. The large range of $\left|V_{C S} / V_{S / C}\right|$ happens for all time periods with both high $(\mathrm{Kp}>4)$ and low $(\mathrm{Kp}<4)$ geomagnetic activities. During periods of low geomagnetic activities, there is still a significant fraction of the events with the ratio $\left|V_{C S} / V_{S / C}\right|$ higher than $10 \%$.

The ST-5 multi-point FAC measurements also allow us to measure the current density using the gradiometry technique pioneered by the 4-spacecraft Cluster mission (Balogh et al. 1997). It is the first mission to provide the necessary multi-point measurements to support magnetic gradiometry in LEO (Slavin et al. 2008). When two spacecraft are within a current sheet simultaneously, the current density can be determined by the gradient of the magnetic field measured at the two spacecraft. This method has the advantage of removing contamination due to temporal variations in the calculation. Temporal variations with wavelengths comparable to or greater than the spacecraft separation (e.g., Alfven waves) are measured simultaneously using the two spacecraft and thus removed in computing the gradients. ST-5 provided numerous opportunities for applying the gradiometry technique when the inter-spacecraft separations went down to $\sim 100 \mathrm{~km}$ or less.

Figure 3 is adapted from Slavin et al. (2008) showing two examples of FAC current density determination by the magnetic grodiometry for auroral oval passes on 15 and 20 June 2006. In ST-5 constellation, the leading, middle and trailing spacecraft in the string of pearl configuration are named 155, 094, and 224, respectively. For the 15 June 2006 auroral oval crossing the 094 - 155 and 094 - 224 spacecraft separations were 131 and $100 \mathrm{~km}$, respectively. The thicknesses of the R1 and R2 intervals were 524 and $442 \mathrm{~km}$, respectively. Similarly, for the 20 June 2006 event, the $094-155$ and 094 - 224 separations were 49 and $92 \mathrm{~km}$, respectively. The thicknesses of the R1 and R2 intervals were 176 and $223 \mathrm{~km}$. Thus in both cases, the spacecraft separations are well below the current sheet thickness. The data from both the leading pair 094 - 155 and the trailing pair $094-224$ can be used to calculate the magnetic gradient. Figure 3 displays the FAC current density using the gradiometry technique for the leading and trailing pairs along with the SC094 single spacecraft, motion-corrected current density. For the 15 June 2006 FAC event (left panel of Fig. 3), all three traces are very similar. Hence, we conclude that for this interval the FACs were very stable over a time span of several minutes. On the other hand, there are differences of up to a factor of 2 between the peak current densities determined from leading and trailing pairs for the 20 June 2006 FAC event. The single spacecraft current density determination from SC094 only agrees well with the values determined by the trailing pair. Since the leading spacecraft 155 was only $\sim 10 \mathrm{~s}$ ahead of the middle spacecraft
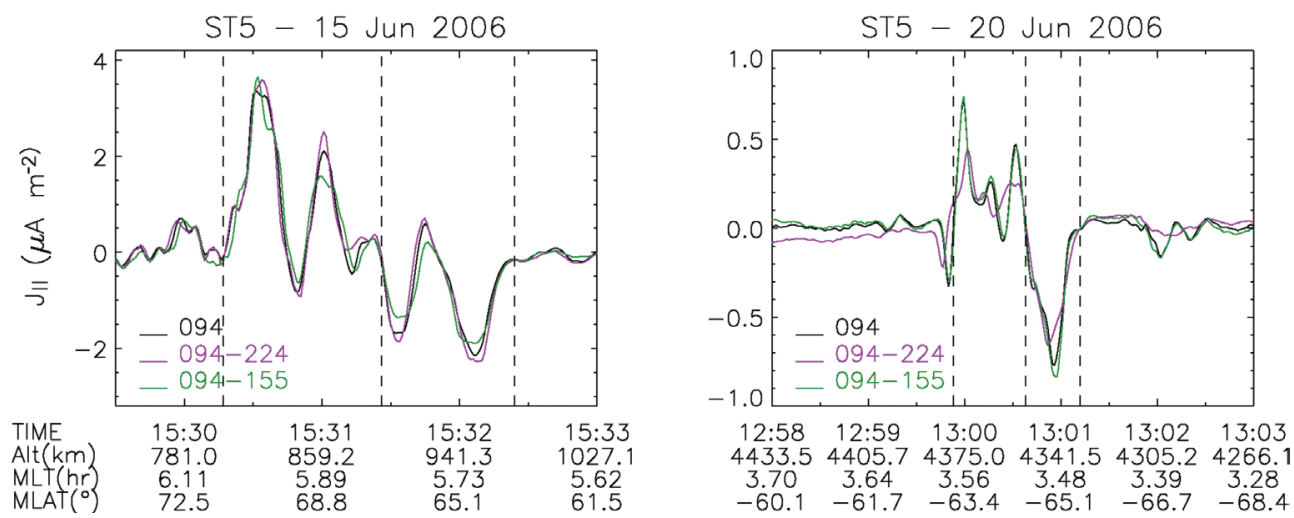

Fig. 3. Two examples of FAC current density determination using the magnetic gradiometry technique. The purple and green traces are from ST-5 pairs 094 - 224 and 094 - 155, respectively. The black trace shows the traditional single spacecraft FAC current determination applied to $12 \mathrm{~s}$ averaged data from SC094 (adapted from Slavin et al. 2008). 
094, there are large, up to $50 \%$ changes took place during this brief interval.

\subsection{Spatial and Temporal Variability of FACs}

FACs are not only in motion but also change with time. Single spacecraft measurements are unable to separate their spatial and temporal variations. Temporal variability in time scales less than $\sim 100 \mathrm{~min}$ (the orbit period of LEO spacecraft) cannot be assessed using data from a single spacecraft. The data from the ST-5 constellation provided the first in situ observations of FAC temporal variability at low altitudes in time scales of $\sim 10 \mathrm{~min}$ and less. As the three spacecraft crossed the FAC region successively along the same trajectory, their magnetic field profiles would track each other exactly with only time delays when the magnetic variations are due to spatial changes. However, any differences in the magnetic field profiles would indicate temporal changes in the current sheet structures. Thus, we can study the tempo- ral variability of the FACs using the magnetic field profiles from multiple spacecraft in a string-of-pearl configuration.

Figure 4 is adapted from Le et al. (2009) showing an overview of the northern and southern polar cap passes during an intense magnetic storm on 14 April 2006. The Dst index at its minimum is $-111 \mathrm{nT}$. The spacecraft trajectories are shown in Fig. 4 (top), in which we mapped the spacecraft positions to their ionospheric footprints at $110 \mathrm{~km}$ altitude along the magnetic field lines. The nested circles represent constant magnetic latitudes separated by $10^{\circ}$ and centered at the Earth's magnetic Pole. (Note we flipped the trajectory for the southern polar cap pass to the northern hemisphere.) The trajectories of the three ST5 spacecraft 094, 155, and 224 are color coded in black, red and blue, respectively. The tick marks on the trajectories are separated by $10 \mathrm{~min}$ with color-coded time tags. During this time period the spacecraft moved across the polar cap near the dawn-dusk meridian plane: from dusk (dawn) to dawn (dusk) in the northern (southern) hemisphere. In the string-of-pearls configuration

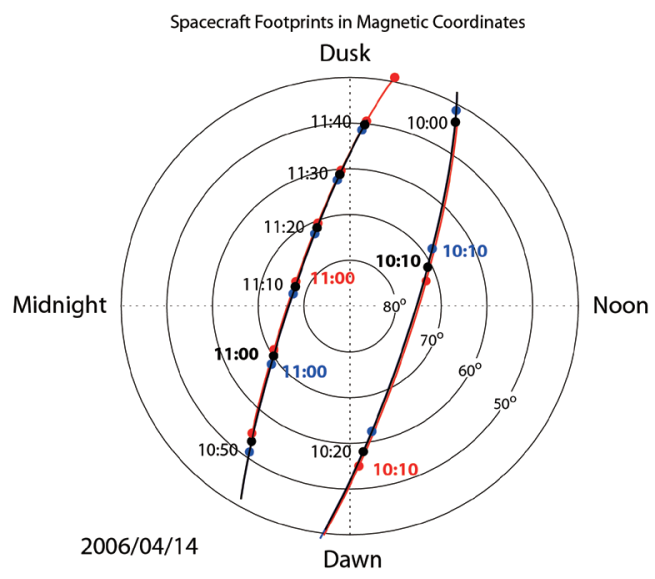

FAC Magnetic Field in SM Coordinate System

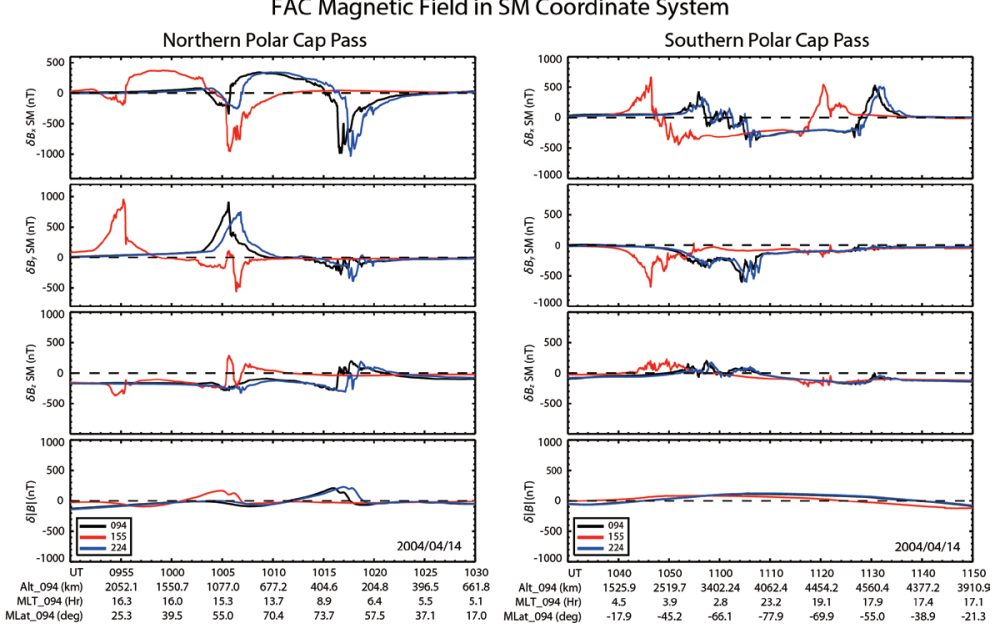

Fig. 4. Overview of FAC observations during the intense magnetic storm on 14 April 2006. (top) The spacecraft ionospheric footprints for a northern and a southern polar cap passes in geomagnetic coordinates. The footprints for the southern pass have been flipped to the northern polar cap. (bottom) Overview of ST5 magnetic field variations generated by the field- aligned currents. The three components of the magnetic field residual vector (data with the internal IGRF model magnetic field removed) are shown in solar magnetic (SM) coordinates. The labels for the spacecraft positions [altitudes, magnetic latitudes, and magnetic local times (MLT)] on the bottom are for mid-spacecraft 094 (black) only (adapted from Le et al. 2009). 
the middle spacecraft 094 and the trailing spacecraft 224 are close together and have a large separation from the leading spacecraft 155 . The lag time along the orbit is about $10 \mathrm{~min}$ for the 094 - 155 pair and about 1 min for the 224 - 094 pair. The spacecraft spacing is $\sim 5331(\sim 3564) \mathrm{km}$ for the $155-094$ pair and $\sim 531(\sim 368) \mathrm{km}$ for the $094-224$ pair over the northern (southern) polar cap. Thus, the observations from this pass allow us to evaluate the FAC variability at these two temporal and spatial scales.

The bottom panels in Fig. 4 show an overview of ST5 magnetic field variations generated by FACs during these two passes, including the three components of the magnetic field residual vector (data with the internal IGRF model magnetic field removed) in the solar magnetic (SM) coordinate system, as well as the residual of the magnetic field strength. The data from the three spacecraft are also color-coded, but the labels for the spacecraft positions [altitudes, magnetic latitudes and magnetic local times (MLT)] are for the middle spacecraft 094 only. Since this was an active period we observed strong FAC activities in the auroral region, on both the dawn and dusk sides, evidenced by perturbations of magnetic field components as large as $\sim 1000 \mathrm{nT}$ in the bottom panels of Fig. 4. Since FACs flow along the background magnetic field direction, their magnetic field perturbations are transverse to the background field with the magnitude much smaller than the background field strength. The presence of FACs only twists the field line direction without changing the field strength, and thus, we do not expect any perturbations in the magnetic field strength. However, we do see some perturbations in the magnetic field strength associated with FACs in the northern polar cap passes near the orbit perigee with altitudes less than $\sim 1000 \mathrm{~km}$, such as in the case in the bottom left panel of Fig. 4. These perturbations in the field strength are generated by strong auroral electrojets in the auroral zone during this active interval. As the altitude increases, their magnetic perturbations decrease and eventually become undetectable, as in the case in the bottom right at altitudes $\sim 3500 \mathrm{~km}$.

In order to examine the temporal variability of FAC structures, Fig. 5 displays the time shifted magnetic field data from the three spacecraft for the 14 April 2006, northern polar cap pass near the perigee, which is in the altitude range of interest for geomagnetism missions. It is also adapted from Le et al. (2009). In this figure, the magnetic field data from the leading (red for SC155) and trailing (blue for SC224) spacecraft are time-shifted to match the middle spacecraft (black for SC094) in order to line up the large-scale current structures observed by the three spacecraft. The time shifts are determined from the cross-correlation analysis of largescale current structures in the 094 - 155 and 094 - 224 pairs. The time labels are on the bottom of the horizontal axis for SC094. The magnetic field components are displayed in the coordinate system determined by the Minimum Variance Analysis (MVA) (Sonnerup and Cahill 1967), where $i, j$, and $k$ are the maximum, intermediate and minimum variance directions, respectively. For FACs, $k$ is also the field-aligned direction and $i$ and $j$ are the two directions transverse to the magnetic field. If the magnetic field variations are seen mainly in one component $\left(\delta B_{i}\right)$, the infinite current sheet would be a good approximation for FACs and the current sheet normal direction would be along the $k$ direction. Otherwise, the magnetic field variations would be seen in both the $i$ and $j$ directions for a current sheet with finite width.

For the duskside FACs (left panel of Fig. 5) large-scale
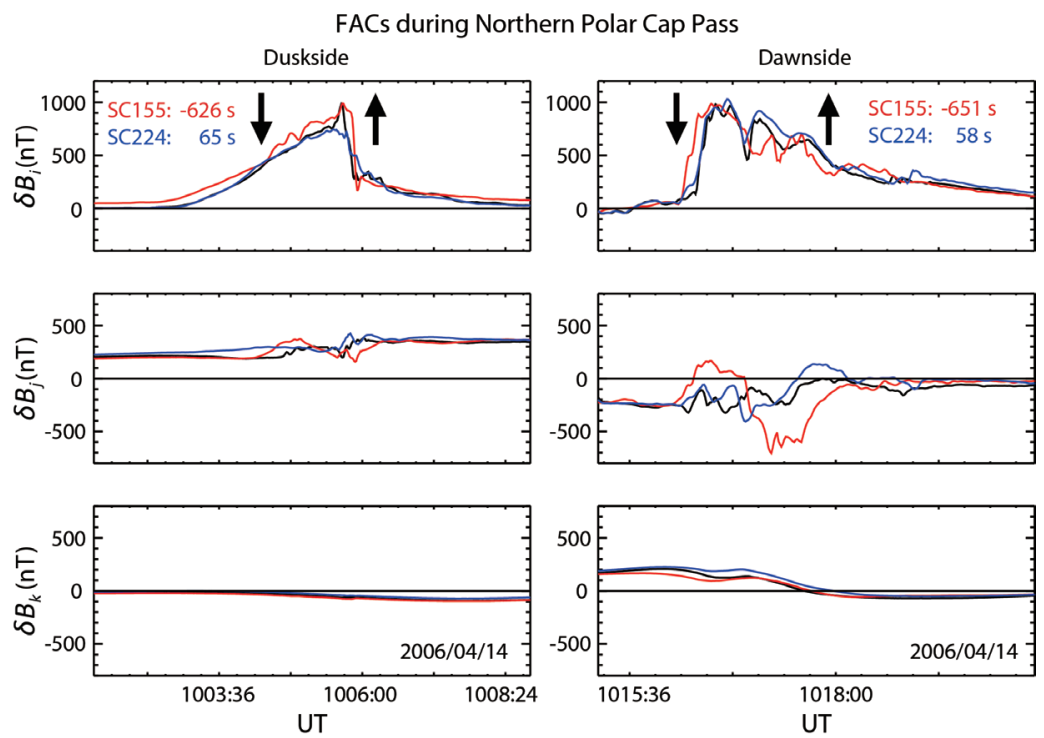

Fig. 5. The time-shifted FAC magnetic field variations displayed in the Minimum Variance Analysis (MVA) coordinate system for the northern polar cap pass during the intense magnetic storm on 14 April 2006. The arrows indicate the general direction of the large-scale currents with a downward arrow for currents flowing into the ionosphere and an upward arrow for currents flowing out of the ionosphere (adapted from Le et al. 2009). 
magnetic field variations are mainly in the $\delta B_{i}$ component, and the $\delta B_{j}$ component contains mainly mesoscale variations with much smaller amplitudes at all three spacecraft. These observations indicate that the infinite current sheet approximation applies to the large-scale FACs observed here. Since the mesoscale variations, which are embedded within the large-scale currents, are seen in both $\delta B_{i}$ and $\delta B_{j}$, the mesoscale currents are filamentary and cannot be treated as infinite sheet currents. For the dawnside FACs in the right panel, the large-scale FACs still appear mainly in the $\delta B_{i}$ component, but there are significant mesoscale variations in the $\delta B_{j}$ component at all three spacecraft. The amplitudes of the $\delta B_{j}$ variations become comparable to those in $\delta B_{i}$ in this case. Clearly the mesoscale currents are generally in the form of current filaments. The traditional method for determining the current density described in Eqs. (1) or (2), which assume an infinite current sheet approximation, will not be applicable to the mesoscale FACs. In this case, calculating the current density requires knowledge of both the $\delta B_{i}$ gradient in the $j$ direction and $\delta B_{j}$ gradient in the $i$ direction. Although the three ST-5 spacecraft in string-of-pearl flight configuration enable us to study the temporal variability of the currents, they are not in the most desirable configuration for measuring the density of these mesoscale currents because they do not provide adequate separations in the eastwest direction (the $i$ direction). It is most desirable that the three spacecraft fly in a triangular configuration in the plane perpendicular to the magnetic field.

From the time-shifted magnetic field data from the three spacecraft in Fig. 5, the FAC temporal variation characteristics are very evident for both FAC intervals. First of all, large-scale currents, shown mainly in $\delta B_{i}$, are relatively stable in time scales of $\sim 10 \mathrm{~min}$. The duration and magnitude of the overall $\delta B_{i}$ variations maintain similar values at the three spacecraft. However, the mesoscale structures embedded within the large-scale currents show significant changes in the same time scales. Comparing the observations of both $\delta B_{i}$ and $\delta B_{j}$ components of $\sim 10 \mathrm{~min}$ apart (red versus black/ blue traces), the mesoscale structures at $\mathrm{SC} 155$ exhibit the largest differences from those of SC094 and SC224. We can observe changes in magnitude, polarity, as well as locations for the mesoscale currents. Meanwhile, the data also show that the time scales for the currents to be relatively stable are $\sim 1 \mathrm{~min}$ for mesoscale currents and at least $\sim 10 \mathrm{~min}$ for large-scale current sheets.

\subsection{Ionospheric Closure of FACs}

Pedersen currents in the ionosphere are the closure currents for FACs. The combined FAC-Pedersen current loops are shown in Fig. 1. Near the dawnside (dusk) auroral oval, R1 FACs flow into (out of) the ionosphere at the high-latitude edge of the oval, while R2 FACs flow out of (into) the ionosphere. Most of the current closure takes place via local
Pedersen currents within the auroral zone flowing between the upward and downward FAC pair, i.e., Pederson currents flow equatorward (poleward) at the dawnside (duskside) auroral zone to form a closed current loop. However, observations show that there is generally an imbalance between the R1 - R2 pair in either dawnside or duskside, i.e., the total current flowing in R1 is more than that in R2 (Iijima and Potemra 1976; Sugiura and Potemra 1976). Thus, there are net currents into (out of) the ionosphere due to the R1 - R2 imbalance in the dawnside (duskside) auroral region. Such net currents need to be closed within the R1 FACs on either side of the pole via cross-polar cap Pedersen currents, also shown in Fig. 1.

We can use a simplified model to calculate the magnetic field perturbations expected from the combined FACPedersen current system. Figure 6 is adapted from Le et al. (2010) showing the FAC current sheet setup and geometry for simple calculations of the magnetic field signatures. The simplified geometry is such that the $X$ direction is from dawn to dusk with the magnetic pole at $\mathrm{X}=0, \mathrm{Z}$ is vertically up along the magnetic pole, and $\mathrm{Y}$ points into the paper, westward (east-ward) in the dawnside (duskside). The infinite planar current sheets are in the YZ plane with current flowing directions shown as arrows in Fig. 6a. The three pairs of balanced current sheets in Fig. 6a (left) are equivalent to the two pairs of unbalanced current sheets in Fig. 6a (right). In Fig. 6b we first calculate the magnetic field from two pairs of balanced R1 - R2 currents on each side of the pole using characteristic current properties listed in the left panel. In this case R1 and R2 are balanced and the net current on either side of the magnetic pole is 0 . The calculated magnetic field in Fig. $6 \mathrm{~b}$ (right) is the well-known unipolar bump in the azimuthal direction (the $\mathrm{Y}$ direction) on either side of the magnetic pole. The east - west component of the magnetic field $\delta B_{y}$ is mainly confined within the R1 - R2 current sheets and quickly decreases to zero away from the current pair, both over the pole and equatorward from the R1 - R2 currents. Next we decrease the current intensity of the R2 current by $25 \%$ so that the R1 - R2 currents are imbalanced, as shown in Fig. 6c (left). The net current flowing into (out of) the ionosphere is $25 \%$ of the total R1 current in the dawnside (duskside). The magnetic field $\delta B_{y}$ within the R1 - R2 circuit remains unipolar with reduced magnitude, as shown in Fig. 6c (right). But there appears to be a magnetic field offset over the pole between the dawnside and duskside FACs. If we further decrease the R2 current intensity so that the net current is $50 \%$ of the total $\mathrm{R} 1$ current, the magnetic field $\delta B_{y}$ offset over the polar cap also increases, as shown in Fig. 6d. Thus, the signature of the imbalanced R1 - R2 pairs is the magnetic field offset over the polar cap. Although the actual FACs and ionospheric current systems are much more complex than this simple model illustrates, it demonstrates the type of magnetic signatures and the magnitudes we expect to observe in situ. Using this offset we 
can quantify the R1 - R2 imbalance based on in situ magnetic field observations from polar-orbiting spacecraft.

Figure 7 displays two examples of ST-5 polar cap crossings showing the magnetic field observations and the deduced current density along the orbit track [adapted from Le et al. (2010)]. The horizontal axis in each panel is the (a)

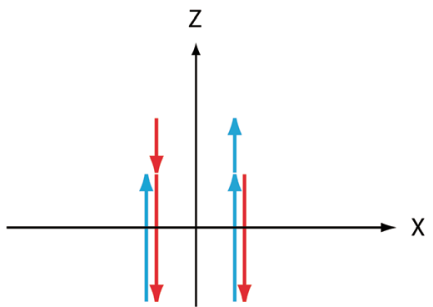

(b)

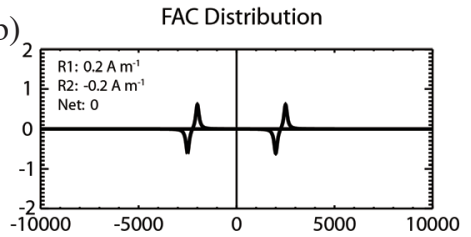

(c)

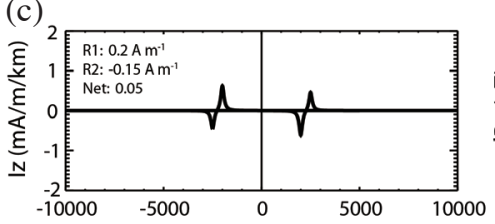

(d)

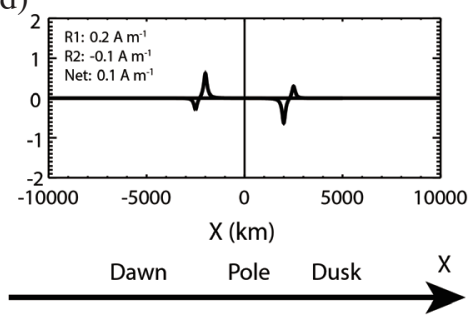

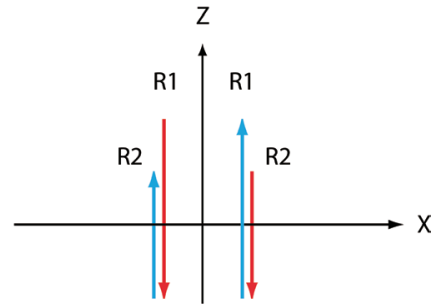
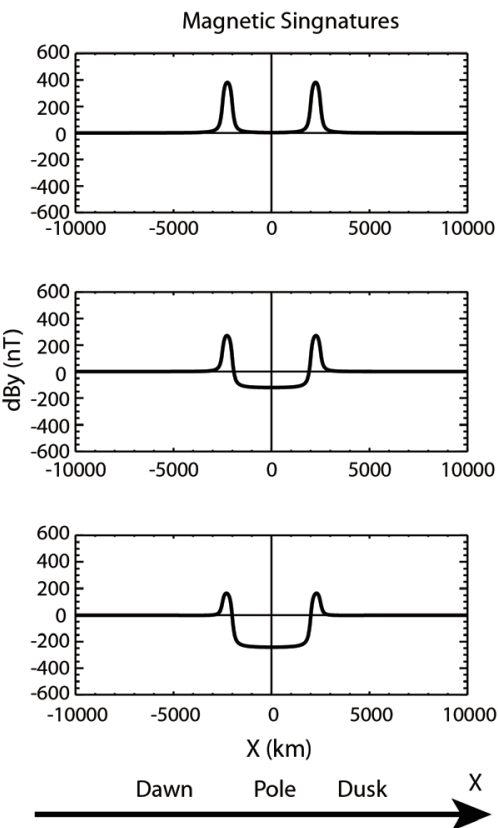

Fig. 6. The FAC current setup and geometry for simple calculations of the magnetic field signatures. (a) Three pairs of (left) balanced infinite current sheets to model the two pairs of (right) unbalanced current sheets. (b - d) Current (left) density distributions and their (right) magnetic field signatures (adapted from Le et al. 2010).
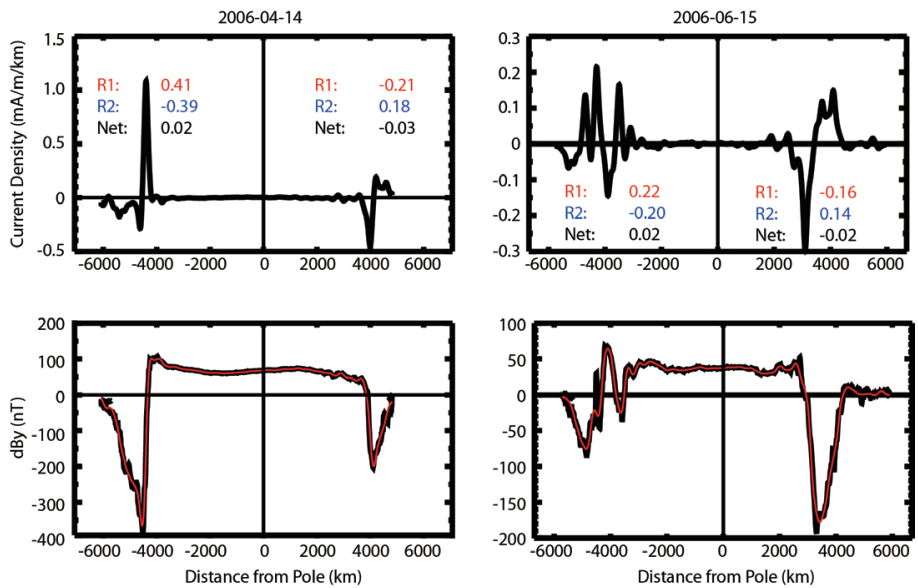

Fig. 7. Two examples of ST-5 polar cap crossings. The bottom panels show the magnetic field signatures of these currents. The top panels show the least-square fit current density distributions along the orbit track. In the bottom panel, the red traces are the best fit magnetic fields, and the black traces are the observed magnetic fields in the cross-track direction along the Y axis (adapted from Le et al. 2010). 
spacecraft distance from the magnetic pole. From both examples, it is very clear that there are indeed magnetic field offsets in $\delta B_{y}$ across the polar cap (bottom panel), indicating that the R1 currents are stronger than the R2 currents and there are net currents flowing into or out of the ionosphere. In order to quantify the imbalance of the R1 - R2 FACs, we calculate the total current intensity using the magnetic field observations for each pass and determine the net current (top panel). Ideally we would like to have two spacecraft, one on either side of the pole, to measure the dawnside and duskside FACs simultaneously. Since the largest time lag of the three ST-5 spacecraft is only $\sim 10 \mathrm{~min}$, we do not have the cases when the dawnside and duskside currents are observed simultaneously. Thus we measure the net current density at the dawnside and duskside individually and examine them statistically. Figure 8 shows a scatter plot of the R2 current intensity versus the R1 current intensity on the duskside and dawnside, respectively, for all the ST- 5 events. In each panel the solid line has a slope that is the average of the R2 intensity to R1 intensity ratio. The dashed line has a slope of 1 , where the R1 and R2 currents have the same intensity. On both the dawnside and dusk side almost all of the data points are located on one side of the dashed line, where the R1 currents are stronger than the $\mathrm{R} 2$ currents. The net currents, due to this R1 - R2 imbalance, are about $5 \%$ of the R1 currents on average on both sides of the pole. This net current will flow as Pedersen current across the polar cap in order to close the imbalanced FACs in the ionosphere. Although the cross-polar cap Pedersen currents are only a small fraction of the R1 currents, they still represent a significant amount of Pedersen currents flowing across the polar cap. Previous observations have determined that the total R1 currents are in the order of a few MA, comparable to the total amount of Chapman-Ferraro current in the magnetopause (e.g., Midgley and Davis Jr. 1963) and the ring current in the inner magnetosphere (e.g., Le et al. 2004). Thus, the total cross-polar cap Pedersen currents are in the order of $\sim 0.1$ MA. Despite the fact that the R1 - R2 imbalance only contributes $~ 5 \%$ of the total R1 currents to the cross-polar cap Pedersen currents whereas $\sim 95 \%$ flow as auroral zone Pedersen currents, the integrated Joule heating rate of the cross-polar cap Pedersen current accounts for a much larger fraction due to the much larger area they flow in the polar cap. Hence, the associated energy dissipation in the polar cap cannot be ignored.

\section{C/NOFS OBSERVATIONS OF THE RING CURRENT DURING MAGNETIC STORMS}

The C/NOFS spacecraft was launched into a nearly circular $13^{\circ}$ inclined orbit on 17 April 2008 with a scientific payload designed to specify and forecast plasma density irregularities in the equatorial ionosphere that degrade transionospheric radio transmissions (de La Beaujardière et al. 2004, 2009). The single satellite is 3 -axis stabilized and has an orbital period of $\sim 97 \mathrm{~min}$. Initial apogee and perigee altitudes were 867 and $401 \mathrm{~km}$, respectively. The Vector Electric Field Instrument (VEFI) suite on the C/NOFS spacecraft includes a sensitive 3-axis fluxgate magnetometer mounted on a 0.6-m boom (Pfaff et al. 2010). Measurements yield full magnetic vectors every second over the range of $\pm 45000 \mathrm{nT}$ with a one-bit resolution of $1.37 \mathrm{nT}$ in each component. During magnetic storms the ring current produces the dominant external magnetic field in the equatorial region. $\mathrm{C} / \mathrm{NOFS}$ provides a complete coverage of all local times every $~ 97 \mathrm{~min}$, a time scale much smaller than the life span of magnetic storms. Thus, C/NOFS magnetic field measurements enable us to study local time variations in the ring current and its evolution during storms. Herein we demonstrate that a single equatorial LEO satellite enables us to monitor and track the ring current evolution, study the local time variation and calculate the near real time Dst index.

In the low-latitude ionosphere the ring current is expected to produce a negative perturbation in the northward magnetic component $\left(\delta B_{N}\right)$. Thus, we concentrate on $\delta B_{N}$ data observed during magnetic storms to examine the ring current characteristics. Figure 9 shows the IMF, the solar wind and the Dst index during the 22 July 2009 magnetic storm, which is one of the events studied in Le et al. (2011). The magnetic storm started shortly after the arrival of an interplanetary shock at 01:00 UT on July 22 . The main phase minimum of $-79 \mathrm{nT}$ in Dst was reached at 09:00 UT. This moderate magnetic storm was a consequence of the strong southward turning of IMF $B_{Z}$ after the shock compression.

Figure 10 shows the local time variations of $\delta B_{N}$ at the six stages of the storm's development corresponding to the vertical dashed lines in Fig. 9. Plots in Fig. 10 use a format similar to that introduced by Love and Gannon (2010). In each panel, a full orbit of $\delta B_{N}$ measurements are displayed as a function of the spacecraft magnetic local time (MLT). The baseline for $\delta B_{N}=0$ is denoted by the dashed line circle. Positive/negative $\delta B_{N}$ is plotted inside/outside the baseline circle. Blue circles represent the Dst index as the radial separations between the blue and baseline circles is the Dst value. Solid black traces represent $\delta B_{N}$ plotted as a function of MLT. Similarly, the radial distance between black traces and baseline circles represent the $\delta B_{N}$ values. Since the C/NOFS orbital plane does not align with the magnetic equator, $\delta B_{N}$ at the spacecraft is normalized by the cosine of the magnetic latitudes where measurements were made. Thus, displayed $\delta B_{N}$ is the component of the magnetic field residual parallel to the geomagnetic dipole axis. The red circle in each panel represents the least square fit to $\delta B_{N}$ using an off-center circle. The circle fitting results in two fitting parameters: the center and the radius of the fitting circle. Small red crosses mark the centers of fitted circles. The center of the fitted circle provides information about the local time asymmetry of $\delta B_{N}$ : (1) its MLT indicates where maximum $\delta B_{N}$ occurs; and (2) its radial displacement from the origin is a measure of the degree of the 

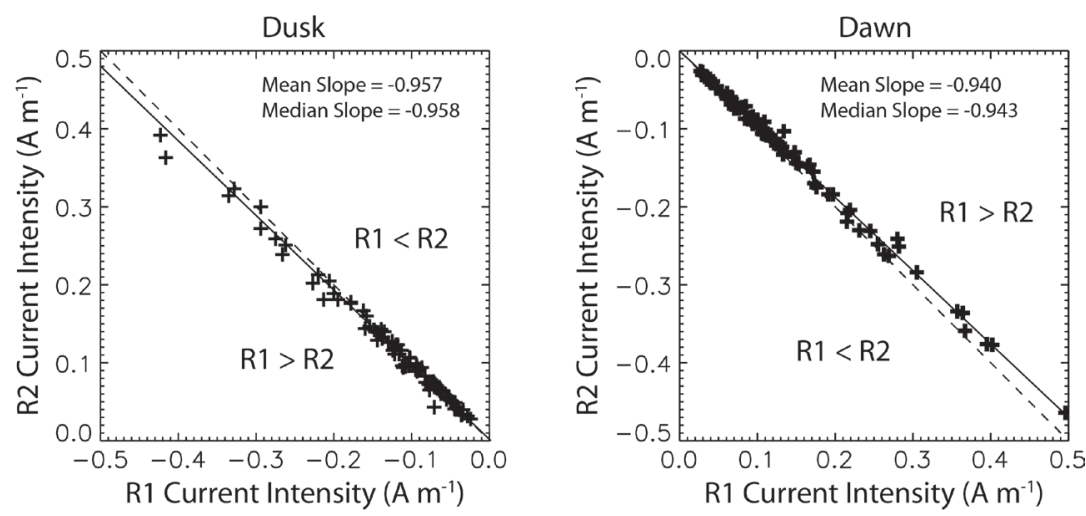

Fig. 8. Statistic results of the R2 versus the R1 current intensity. The solid line has a slope, which is the average of the R2 - R1 intensity ratio. The dashed line has a slope of 1 (adapted from Le et al. 2010).

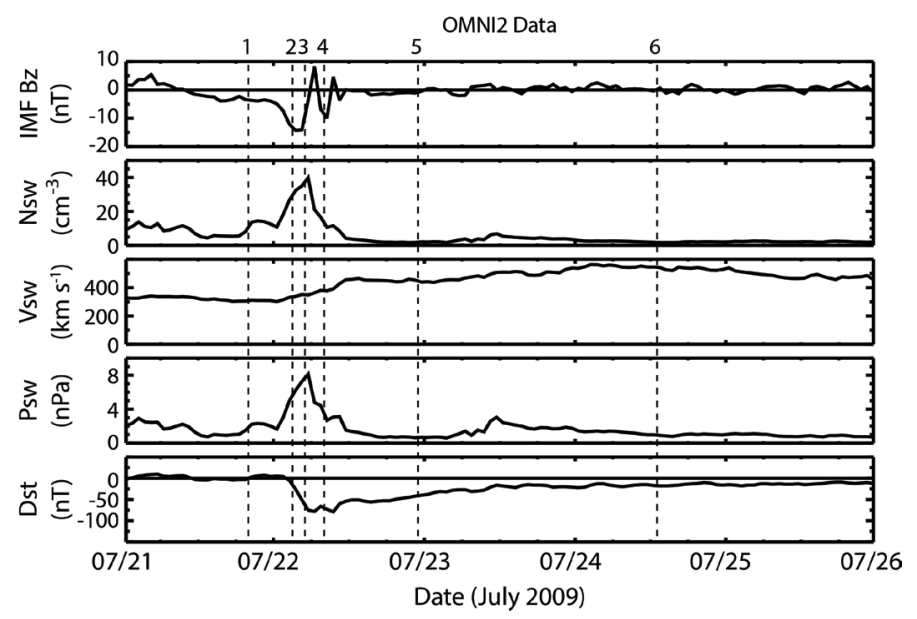

Fig. 9. Overview of the 22 July 2009 storm containing 5 days of hourly averaged OMNI data from 21 - 25 July 2009. Shown from top to bottom are the interplanetary magnetic field (IMF) Bz component, the solar wind density, velocity, dynamic pressure, and the Kyoto provisional Dst index (adapted from Le et al. 2011).

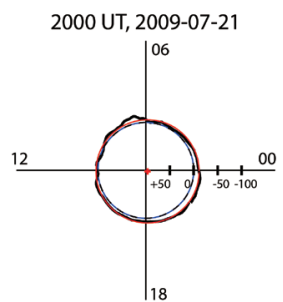

08:00 UT, 2009-07-22

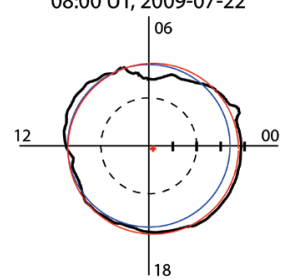

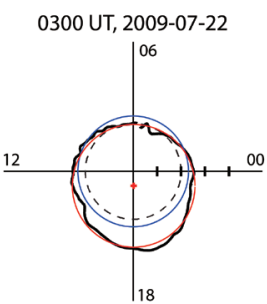

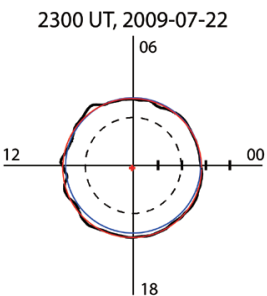

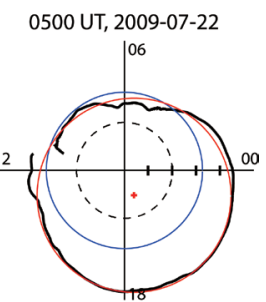

1300 UT, 2009-07-24

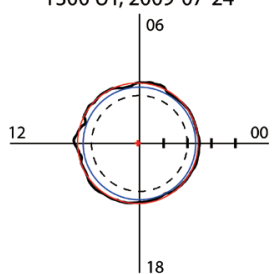

Fig. 10. Local time variations of $\mathrm{dB}_{\mathrm{N}}$ at various stages of the storm evolution, corresponding to the times marked by the dashed lines in Fig. 10 (adapted from Le et al. 2011). 
MLT asymmetry of $\delta B_{N}$. Radii of fitted circles (after $100 \mathrm{nT}$ baseline removal) are nearly identical to the absolute value of the orbital-averaged $\delta B_{N}$. As an analogy to how the Dst index is estimated from the ground-based $\delta B_{N}$, the orbital-averaged $\delta B_{N}$ from C/NOFS data can be used as a real-time provisional Dst index. We note that the equatorial electrojet (EEJ) also contributes to equatorial $\delta B_{N}$ in the dayside. On the ground its signal reaches up to $80 \mathrm{nT}$ near the dayside magnetic equator (Manoj et al. 2006) and their effect is avoided by using midlatitude ground stations in the Dst index calculation. Spacecraft observations show that EEJ signals are confined mainly within $\pm 3^{\circ}$ from the magnetic equator and maximize between 10:30 - 12:00 LT; and their $\delta B_{N}$ magnitudes are in the order of $20 \mathrm{nT}$ at $\sim 450 \mathrm{~km}$ and $\sim 10 \mathrm{nT}$ at $\sim 700 \mathrm{~km}$ (Alken and Maus 2007). Thus, the EEJ magnetic signals near the magnetic equator are in the same order of the quiet time ring current in typical ionospheric satellite altitudes of $\sim 400-700 \mathrm{~km}$ range, and much smaller than those of stormtime ring current. Here we ignored the EEJ effect in the study of the stormtime ring current as the spacecraft is outside the EEJ region for most of the orbit.

Figure 10 provides a basis for perceiving the morphology of the ring current's local time evolution. Panel 1 describes the pre-storm situation when the Dst and $\delta B_{N}$ traces were very close to the baseline. Even in very quiet times the red cross centroid was slightly shifted $(4.1 \mathrm{nT})$ from the origin toward the pre-midnight sector, indicating the ring current is slightly asymmetric. Panels 2 and 3 show $\delta B_{N}$ distributions measured during the early main phase and at maximum epoch, when the centroid was shifted toward the duskevening MLT sector by 30.2 and $55.6 \mathrm{nT}$, respectively. This is a sign that the storm time ring current quickly becomes very asymmetric during the main phase. Comparing the red and blue circles we see that near the dawn meridian (where the minimum $\delta B_{N}$ occurs) Dst was slightly more negative than $\delta B_{N}$. However, similar to DMSP observations (Burke et al. 2011), at evening- midnight local times $\delta B_{N}$ was signifi- cantly more negative than Dst. This asymmetry is contributed by the rapid development of a partial ring current as well as the remote FACs that close the partial ring current in the ionosphere. The maximum of $\delta B_{N}$ is in the evening-midnight section during the main phase. Panel 4 shows the near early recovery phase and a slight Dst dip to a second minimum the $\delta B_{N}$ distribution appears to be far more symmetrical than was detected during the two previous orbits. The red centroid displacement moved back to $10.9 \mathrm{nT}$. Thus, the ring current recovery in this case started with a rapid decay of the partial ring current. Panels 5 and 6 indicate that during the later parts of the recovery phase the Dst and $\delta B_{N}$ distribution traces come closer together suggesting that the ring current approached, but did not fully achieve, exact symmetry.

We compare real-time Dst with the orbit-averaged $\delta B_{N}$ for this storm, as shown in Fig. 11. The right panel shows real-time Dst (the red line) and the orbit-averaged $\delta B_{N}$ (black stars) plotted as functions of UT across the entire storm interval. The left panel contains a scatter plot of orbit-averaged $\delta B_{N}$ versus Dst. Superposed on the plot are the numerical and graphic (red line) results of linear regression analyses performed on the plotted data. The dashed line with a slope of unity is provided for reference. For this case the linear regression slope is near unity (0.977) and the correlation coefficient is very high $(0.970)$. It is also clear that the orbitaveraged $\delta B_{N}$ data points generally fell below Dst traces. This was most prevalent near storm time maximum epochs. It is also reflected in the $-9.7 \mathrm{nT}$ intercept obtained through linear regression analysis. There are two reasons for the baseline differences. First, the real-time Dst has known offsets from final Dst index. Second, such a difference is expected even with final Dst since the Dst does not consider stable magnetospheric fields such as the $8 \mathrm{nT}$ from the magnetotail currents and a few $\mathrm{nT}$ from the quiet time ring current (e.g., Lühr and Maus 2010). This example along with the others in Le et al. (2011) demonstrate that we can extract a parameter $\delta B_{N}$ whose orbit-averaged characteristics mimic those of the
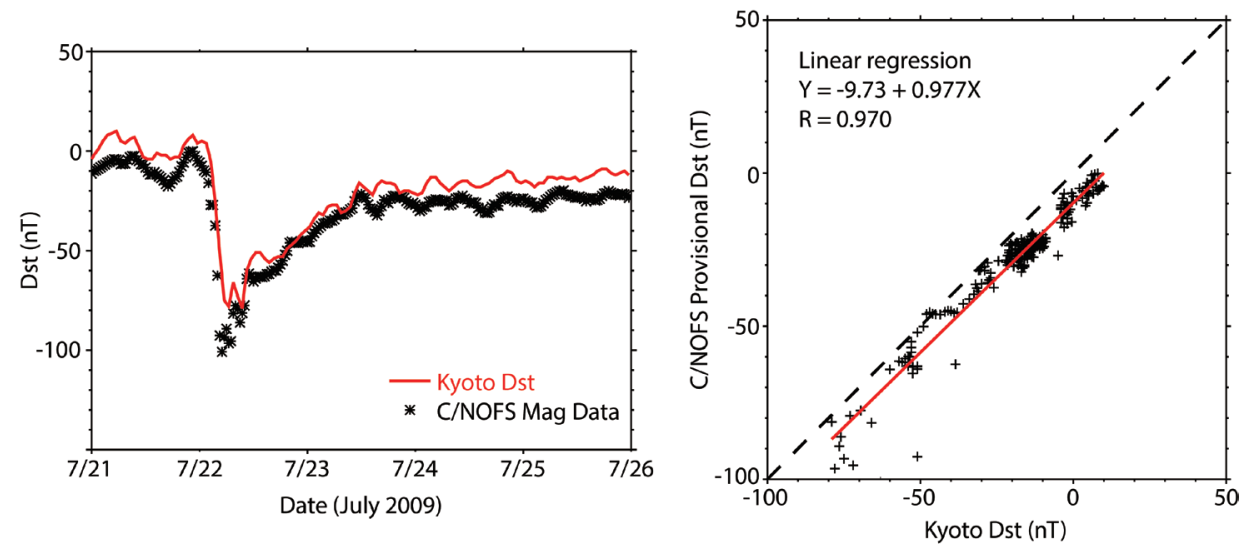

Fig. 11. Comparison between the Kyoto Dst index and the provisional Dst index determined from the C/NOFS magnetic field data for the 22 July 2009 magnetic storm (adapted from Le et al. 2011). 
provisional Dst index, an important input parameter for geomagnetic modeling

\section{CONCLUDING REMARKS}

The external currents driven by the solar wind-magnetosphere interaction are very dynamic and change in various time scales that are much shorter than those of internal sources. The external currents are primarily ordered by the local time, which is very different from the Earth's internal field. We presented recent observations of the Earth's magnetic field from LEO satellites, including polar orbiting ST-5 spacecraft and low-inclination C/NOFS spacecraft, demonstrating that data from multiple spacecraft are required to characterize these external currents. Based on our recent observations as well as previous work in the literature, we summarize the findings based on these measurements:

(1) Simultaneous multi-point measurements along a single LEO polar orbit can reveal the temporal variability of FACs in various time scales, measure the motion of large scale current sheets, provide opportunities for magnetic gradiometer determination of the current density, quantify the closure path of ionospheric Pedersen current and assess the strength of auroral electrojets.

(2) Measurements from a single equatorial LEO satellite can specify the ring current's temporal evolution, quantify its local time asymmetry and extract a timely proxy for the provisional Dst index at high cadence.

(3) FACs have very complex structures with filamentary currents in various scales embedded within large-scale current sheets. Simultaneous measurements with longitudinal separations less than $\sim 500 \mathrm{~km}$ are also required to specify their meso-scale variations.

(4) Simultaneous monitoring of the dawn-dusk, day-night, and north-south auroral zones are also needed to specify the global distribution of FACs and ionospheric currents. This requires placing multiple satellites in polar orbits with large local time separations $(\sim 3-6 \mathrm{~h})$.

Great advances have been made in geomagnetism investigations since space-based magnetic field measurements from dedicated geomagnetism satellites (Magsat, Orsted, and CHAMP) became available. The Swarm mission will be the first geomagnetism constellation (Friis-Christensen et al. 2006). It contains three satellites, two at lower altitude flying side-by-side and one at higher altitude slowly drifting away from the lower-altitude pair longitudinally. It will return the first simultaneous geomagnetism measurements at different latitudes and longitudes. For post-Swarm geomagnetism satellite missions, it is desirable to have a constellation of more than three satellites in a combination of both low and high inclination orbits. The constellation would provide simultaneous measurements not only at different latitudes and local times, but also with a global coverage. These measurements will result in a global specification of the external currents and enable us to separate their magnetic contributions from the main field measurements.

In the post-Swarm era a desired geomagnetism constellation mission would contain both low and high inclination satellites. From the point of view optimal for measuring the external currents, the low-inclination satellites would be dedicated to the low-latitude current systems (the ring current, the magnetopause current and the tail current), while highinclination satellites to the high-latitude current systems (the combined field-aligned/Pedersen currents and auroral electrojets). Based on our recent observations, we would recommend the following constellation configuration:

(1) Two or three satellites in the same polar orbit to measure FACs and their temporal variability.

(2) Additional two or three spacecraft in polar orbits, equally spaced in local time among all the polar orbits, to provide the global coverage of magnetospheric and ionospheric currents.

(3) One satellite in a low-inclination orbit to monitor the symmetric and asymmetric parts of the ring current.

Such a constellation is able to provide unprecedented geomagnetism data set with simultaneous measurements of current systems at various temporal and spatial scales, and simultaneous measurements in both in northern and southern polar regions, with high accuracy and high precision measurements with repeated paths. It allows quantitatively distinguishing the external effects from the main internal field at a time scale shorter than an orbit period of LEO satellites.

Acknowledgements G. Le would like to thank the co-authors of the previous work used in this review, in particular Yongli Wang and Robert Strangeway for their help with the analysis of ST-5 data, and William Burke, Henry Freudenreich, Stefan Maus, and Hermann Luhr for their help with the analysis of C/NOFS data. We would like to thank all of the people who made the ST-5 and C/NOFS missions successful. The ST-5 mission was a project at NASA Goddard Space Flight Center. The C/NOFS mission was made possible by Air Force Research Laboratory Space Vehicles Directorate. The Wind solar wind and IMF data and the Dst index used in this study are extracted from the CDAWeb and OMNIWeb, respectively. The Dst index is calculated at Kyoto University.

\section{REFERENCES}

Alken, P. and S. Maus, 2007: Spatio-temporal characterization of the equatorial electrojet from CHAMP, Ørsted, and SAC-C satellite magnetic measurements. J. Geophys. Res., 112, A09305, doi: 10.1029/2007JA012524. [Link]

Balogh, A., M. W. Dunlop, S. W. H. Cowley, D. J. Southwood, J. G. Thomlinson, K. H. Glassmeier, G. Musmann, H. Lühr, S. Buchert, M. H. Acuña, D. H. 
Fairfield, J. A. Slavin, W. Riedler, K. Schwingenschuh, and M. G. Kivelson, 1997: The Cluster magnetic field investigation. Space Sci. Rev., 79, 65-91, doi: 10.1023/A:1004970907748. [Link]

Burke, W. J., G. R. Wilson, C. S. Lin, F. J. Rich, J. O. Wise, and M. P. Hagan, 2011: Estimating Dst indices and exospheric temperatures from equatorial magnetic fields measured by DMSP satellites. J. Geophys. Res., 116, A01205, doi: 10.1029/2010JA015310. [Link]

Burton, R. K., R. L. McPherron, and C. T. Russell, 1975: An empirical relationship between interplanetary conditions and Dst. J. Geophys. Res., 80, 4204-4214, doi: 10.1029/JA080i031p04204. [Link]

Cargill, P. J., M. W. Dunlop, A. Balogh, and the FGM Team, 2001: First Cluster results of the magnetic field structure of the mid- and high-altitude cusps. Ann. Geophys., 19, 1533-1543, doi: 10.5194/angeo-19-1533-2001. [Link]

Cowley, S. W. H., 1984: Solar wind control of magnetospheric convection. In: Battrick, B. T. and E. Rolfe (Eds.), Achievements Of The International Magnetospheric Study (IMS), ESA Scientific \& Technical Publications, Noordwijk, Netherlands, 483-494.

De La Beaujardière, O., L. Jeong, B. Basu, S. Basu, T. Beach, P. Bernhardt, W. Burke, K. Groves, R. Heelis, R. Holzworth, C. Huang, D. Hunton, M. Kelley, R. Pfaff, J. Retterer, F. Rich, M. Starks, P. Straus, and C. Valladares, 2004: C/NOFS: A mission to forecast scintillations. J. Atmos. Sol.-Terr. Phys., 66, 1573-1591, doi: 10.1016/j.jastp.2004.07.030. [Link]

De La Beaujardière, O., J. M. Retterer, R. F. Pfaff, P. A. Roddy, C. Roth, W. J. Burke, Y. J. Su, M. C. Kelley, R. R. Ilma, G. R. Wilson, L. C. Gentile, D. E. Hunton, and D. L. Cooke, 2009: C/NOFS observations of deep plasma depletions at dawn. Geophys. Res. Lett., 36, L00C06, doi: 10.1029/2009GL038884. [Link]

Dessler, A. J. and E. N. Parker, 1959: Hydromagnetic theory of geomagnetic storms. J. Geophys. Res., 64, 22392252, doi: 10.1029/JZ064i012p02239. [Link]

Dessler, A. J., W. E. Francis, and E. N. Parker, 1960: Geomagnetic storm sudden-commencement rise times. $J$. Geophys. Res., 65, 2715-2719, doi: 10.1029/JZ065i009p02715. [Link]

Draper, N. C., M. Lester, S. W. H. Cowley, J. A. Wild, S. E. Milan, G. Provan, A. Grocott, A. N. Fazakerley, A. Lahiff, J. A. Davies, J. M. Bosqued, J. P. Dewhurst, R. Nakamura, C. J. Owen, J. Waterman, M. G. Henderson, H. J. Singer, and E. Donovan, 2005: Cluster magnetotail observations of a tailward-travelling plasmoid at substorm expansion phase onset and field aligned currents in the plasma sheet boundary layer. Ann. Geophys., 23, 3667-3683, doi: 10.5194/angeo-23-3667-2005. [Link]

Echer, E., W. D. Gonzalez, and B. T. Tsurutani, 2008: Interplanetary conditions leading to superintense geomagnetic storms (Dst $\leq-250 \mathrm{nT}$ ) during solar cycle 23. Geophys.
Res. Lett., 35, L06S03, doi: 10.1029/2007GL031755. [Link]

Figueiredo, S., G. T. Marklund, T. Karlsson, T. Johansson, Y. Ebihara, M. Ejiri, N. Ivchenko, P. A. Lindqvist, H. Nilsson, and A. Fazakerley, 2005: Temporal and spatial evolution of discrete auroral arcs as seen by Cluster. Ann. Geophys., 23, 2531-2557, doi: 10.5194/ angeo-23-2531-2005. [Link]

Friis-Christensen, E., Y. Kamide, A. D. Richmond, and S. Matsushita, 1985: Interplanetary magnetic field control of high-latitude electric fields and currents determined from Greenland Magnetometer Data. J. Geophys. Res., 90, 1325-1338, doi: 10.1029/JA090iA02p01325. [Link]

Friis-Christensen, E., H. Lühr, and G. Hulot, 2006: Swarm: A constellation to study the Earth's magnetic field. Earth Planets Space, 58, 351-358, doi: 10.1186/ BF03351933. [Link]

Fukushima, N. and Y. Kamide, 1973: Partial ring current models for worldwide geomagnetic disturbances. Rev. Geophys., 11, 795-853, doi: 10.1029/ RG011i004p00795. [Link]

Glatzmaier, G. A. and P. H. Roberts, 1995a: A three-dimensional convective dynamo solution with rotating and finitely conducting inner core and mantle. Phys. Earth Planet. Inter., 91, 63-75, doi: 10.1016/0031-9201(95)03049-3. [Link]

Glatzmaier, G. A. and P. H. Roberts, 1995b: A three-dimensional self-consistent computer simulation of a geomagnetic field reversal. Nature, 377, 203-209, doi: 10.1038/377203a0. [Link]

Greenspan, M. E. and D. C. Hamilton, 2000: A test of the Dessler-Parker-Sckopke relation during magnetic storms. J. Geophys. Res., 105, 5419-5430, doi: 10.1029/1999JA000284. [Link]

Iijima, T. and T. A. Potemra: 1976: The amplitude distribution of field-aligned currents at northern high latitudes observed by Triad. J. Geophys. Res., 81, 2165-2174, doi: 10.1029/JA081i013p02165. [Link]

Iijima, T. and T. A. Potemra,1978: Large-scale characteristics of field-aligned currents associated with substorms. J. Geophys. Res., 83, 599-615, doi: 10.1029/ JA083iA02p00599. [Link]

Iyemori, T., 2000: Formation of the storm-time ring current and the Dst field: Some recent topics. In: Ohtani, S. I., R. Fujii, M. Hesse, and R. L. Lysak (Eds.), Magnetospheric Current Systems, Geophysical Monograph, Vol. 118, American Geophysical Union, Washington D. C., 331-338, doi: 10.1029/GM118p0331. [Link]

Iyemori, T., T. Araki, T. Kamei, and M. Takeda, 1992: Midlatitude geomagnetic indices ASY and SYM (Provisional) No. 1, 1989, Data Anal. Center for Geomagn. and Space Magn., Kyoto Univ., Kyoto, Japan.

Johansson, T., S. Figueiredo, T. Karlsson, G. Marklund, A. 
Fazakerley, S. Buchert, P. A. Lindqvist, and H. Nilsson, 2004: Intense high-altitude auroral electric fields temporal and spatial characteristics. Ann. Geophys., 22, 2485-2495, doi: 10.5194/angeo-22-2485-2004. [Link]

Kamide, Y., A. D. Richmond, and S. Matsushita, 1981: Estimation of ionospheric electric fields, ionospheric currents, and field-aligned currents from ground magnetic records. J. Geophys. Res., 86, 801-813, doi: 10.1029/ JA086iA02p00801. [Link]

Le, G., C. T. Russell, and K. Takahashi, 2004: Morphology of the ring current derived from magnetic field observations. Ann. Geophys., 22, 1267-1295, doi: 10.5194/ angeo-22-1267-2004. [Link]

Le, G., Y. Wang, J. A. Slavin, and R. J. Strangeway, 2009: Space Technology 5 multipointobservations of temporal and spatial variability of field-aligned currents. J. Geophys. Res., 114, A08206, doi: 10.1029/2009JA014081. [Link]

Le, G., J. A. Slavin, and R. J. Strangeway, 2010: Space Technology 5 observations of the imbalance of regions 1 and 2 field-aligned currents and its implication to the cross-polar cap Pedersen currents. J. Geophys. Res., 115, A07202, doi: 10.1029/2009JA014979. [Link]

Le, G., W. J. Burke, R. F. Pfaff, H. Freudenreich, S. Maus, and H. Lühr, 2011: C/NOFS measurements of magnetic perturbations in the low-latitude ionosphere during magnetic storms. J. Geophys. Res., 116, A12230, doi: 10.1029/2011JA017026. [Link]

Love, J. J. and J. L. Gannon, 2010: Movie-maps of lowlatitude magnetic storm disturbance. Space Weather, 8, S06001.

Lühr, H. and S. Maus, 2010: Solar cycle dependence of quiet-time magnetospheric currents and a model of their near-Earth magnetic fields. Earth Planets Space, 62, 843-848, doi: 10.5047/eps.2010.07.012. [Link]

McPherron, R. L., C. T. Russell, and M. P. Aubry, 1973: Satellite studies of magnetospheric substorms on August 15, 1968: 9. Phenomenological model for substorms. J. Geophys. Res., 78, 3131-3149, doi: 10.1029/ JA078i016p03131. [Link]

Midgley, J. E. and L. Davis Jr., 1963: Calculation by a moment technique of the perturbation of the geomagnetic field by the solar wind. J. Geophys. Res., 68, 51115123, doi: 10.1029/JZ068i018p05111. [Link]

Manoj, C., H. Lühr, S. Maus, and N. Nagarajan, 2006: Evidence for short spatial correlation lengths of the noontime equatorial electrojet inferred from a comparison of satellite and ground magnetic data.J. Geophys. Res., 111, A11312, doi: 10.1029/2006JA011855. [Link]

Moretto, T., N. Olsen, P. Ritter, and G. Lu, 2002: Investigating the auroral electrojets with low altitude polar orbiting satellites. Ann. Geophys., 20, 1049-1061, doi: 10.5194/angeo-20-1049-2002. [Link]
Ohtani, S., M. Nosé, G. Rostoker, H. Singer, A. T. Y. Lui, and M. Nakamura, 2001: Storm-substorm relationship: Contribution of the tail current to Dst. J. Geophys. Res., 106, 21199-21209, doi: 10.1029/2000JA000400. [Link]

Olsen, N. and C. Stolle, 2012: Satellite Geomagnetism. Annu. Rev. Earth Planet. Sci., 40, 441-465, doi: 10.1146/annurev-earth-042711-105540. [Link]

Pfaff, R., D. Rowland, H. Freudenreich, K. Bromund, G. Le, M. Acuña, J. Klenzing, C. Liebrecht, S. Martin, W. J. Burke, N. C. Maynard, D. E. Hunton, P. A. Roddy, J. O. Ballenthin, and G. R. Wilson, 2010: Observations of DC electric fields in the low-latitude ionosphere and their variations with local time, longitude, and plasma density during extreme solar minimum. J. Geophys. Res., 115, A12324, doi: 10.1029/2010JA016023. [Link]

Sckopke, N., 1966: A general relation between the energy of trapped particles and the disturbance field near the Earth. J. Geophys. Res., 71, 3125-3130, doi: 10.1029/ JZ071i013p03125. [Link]

Slavin, J. A., G. Le, R. J. Strangeway, Y. Wang, S. A. Boardsen, M. B. Moldwin, and H. E. Spence, 2008: Space Technology 5 multi-point measurements of near-Earth magnetic fields: Initial results. Geophys. Res. Lett., 35, L02107, doi: 10.1029/2007GL031728. [Link]

Sonnerup, B. U. Ö. and L. J. Cahill Jr., 1967: Magnetopause structure and attitude from Explorer 12 observations. J. Geophys. Res., 72, 171-183, doi: 10.1029/ JZ072i001p00171. [Link]

Sugiura, M. and T. A. Potemra, 1976: Net field-aligned currents observed by Triad. J. Geophys. Res., 81, 21552164, doi: 10.1029/JA081i013p02155. [Link]

Turner, N. E., D. N. Baker, T. I. Pulkkinen, and R. L. McPherron, 2000: Evaluation of the tail current contribution to Dst. J. Geophys. Res., 105, 5431-5439, doi: 10.1029/1999JA000248. [Link]

Turner, N. E., D. N. Baker, T. I. Pulkkinen, J. L. Roeder, J. F. Fennell, and V. K. Jordanova, 2001: Energy content in the storm time ring current. J. Geophys. Res., 106, 19149-19156, doi: 10.1029/2000JA003025. [Link]

Wang, Y., G. Le, J. A. Slavin, S. A. Boardsen, and R. J. Strangeway, 2009: Space Technology 5 measurements of auroral field-aligned current sheet motion. Geophys. Res. Lett., 36, L02105, doi: 10.1029/2008GL035986. [Link]

Zanetti, L. J., W. Baumjohann, T. A. Potemra, and P. F. Bythrow, 1984: Three-dimensional Birkeland-ionospheric current system, determined from MAGSAT. In: Zanetti, L. J., W. Baumjohann, T. A. Potemra, and P. F. Bythrow (Eds.), Magnetospheric Currents, Geophysical Monograph Series, Vol. 28, American Geophysical Union, Washington D. C. , 123-130, doi: 10.1029/GM028p0123. [Link] 